\title{
Concurrent Subcutaneous Candidal Abscesses and Pulmonary Cryptococcosis in a Patient with Diabetes Mellitus and a History of Corticosteroid Therapy
}

\author{
Hiroshi Kakeya ${ }^{1,2}$, Koichi Izumikawa ${ }^{2}$, Koichi Yamada ${ }^{1,2}$, Yusuke Narita ${ }^{2}$, Tomoya Nishino ${ }^{2}$, \\ Yoko Obata ${ }^{2}$, Takahiro Takazono ${ }^{2}$, Shintaro Kurihara ${ }^{3}$, Kosuke Kosai ${ }^{3}$, \\ Yoshitomo Morinaga ${ }^{4}$, Shigeki Nakamura ${ }^{2}$, Yoshifumi Imamura ${ }^{2}$, Taiga Miyazaki ${ }^{2}$, \\ Misuzu Tsukamoto ${ }^{3}$, Katsunori Yanagihara ${ }^{4}$, Motoi Takenaka ${ }^{5}$, \\ Takayoshi Tashiro $^{6}$ and Shigeru Kohno ${ }^{2}$
}

\begin{abstract}
A 50-year-old man with a history of long-term corticosteroid treatment following adrenalectomy for Cushing's syndrome and uncontrolled diabetes mellitus was admitted for an examination of an abnormal thoracic shadow. Cryptococcal serum antigens were positive, and the histopathology of a lung biopsy showed encapsulated yeast resembling Cryptococcus neoformans. On admission, the serum $\beta$-D-glucan level was approximately twice the cutoff value, several nodules were observed on both legs and magnetic resonance imaging revealed subcutaneous abscesses. Candida albicans was identified from needle aspirates, and the patient was successfully treated with fluconazole and flucytosine. We herein report the first case of concurrent $C$. albicans skin abscesses and pulmonary cryptococcosis.
\end{abstract}

Key words: subcutaneous candidal abscess, pulmonary cryptococcosis, serum 1,3- $\beta$-D-glucan

(Intern Med 53: 1385-1390, 2014)

(DOI: 10.2169/internalmedicine.53.1409)

\section{Introduction}

Cryptococcus neoformans is a ubiquitous, encapsulated, yeast-like fungus found worldwide, particularly in soils that are contaminated with pigeon droppings and decaying wood. Pulmonary cryptococcosis is caused by the inhalation of $\mathrm{Cr}$. neoformans into the lungs, with subsequent hematogenous dissemination that may induce central nervous system infection. Although pulmonary cryptococcosis can occur in both healthy individuals and immunocompromised patients, it is frequently recognized as an opportunistic pathogen, particularly in patients with lymphohematological disorders, those receiving steroids or immunosuppressants and those with acquired immunodeficiency syndrome (AIDS) $(1,2)$. Another Cryptococcus subspecies, Cr. gattii has been cultured from river red gum trees (Eucalyptus camaldulensis) and forest red gum trees (Eucalyptus tereticornis) in Australia (3, 4). In addition, an outbreak of $\mathrm{Cr}$. gattii infection was reported in Vancouver Island, British Columbia in 1999 (5).

Candida albicans is the most common cause of candidiasis; however, there has been an increase in the isolation of non-albicans Candida species (i.e., C. glabrata, C. parapsilosis, C. tropicalis and C. krusei) in recent years $(6,7)$.

The clinical manifestations of candidiasis range from local mucosal membrane infection to widespread dissemina-

${ }^{1}$ Department of Infection Control Science, Graduate School of Medicine, Osaka City University, Japan, ${ }^{2}$ Department of Molecular Microbiology and Immunology, Nagasaki University Graduate School of Biomedical Sciences, Japan, ${ }^{3}$ Nagasaki University Infection Control and Education Center, Nagasaki University Hospital, Japan, ${ }^{4}$ Department of Laboratory Medicine, Nagasaki University Hospital, Japan, ${ }^{5}$ Department of Dermatology, Nagasaki University Graduate School of Biomedical Sciences, Japan and ${ }^{6}$ Department of Health Sciences, Nagasaki University Graduate School of Biomedical Sciences, Japan

Received for publication July 20, 2013; Accepted for publication January 5, 2014

Correspondence to Dr. Hiroshi Kakeya, kakeya-ngs@umin.ac.jp 


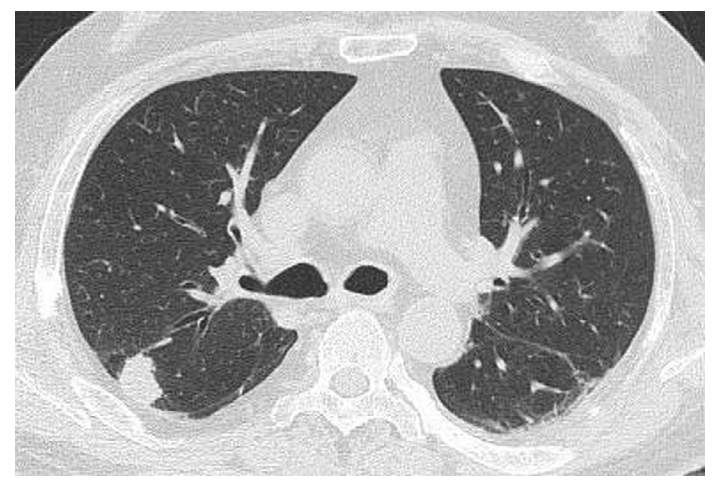

Figure 1. Thoracic computed tomography (CT) image obtained on admission showing a solitary well-defined, pleuralbased nodule in the right $\mathrm{S} 2$ region. No pleural effusion or cavitation were observed.

tion. Local overgrowth on mucous membranes (oropharyngeal involvement or vaginitis) is often observed in patients with changes in normal flora or deficiencies in cell-mediated immunity, as in AIDS. Invasive focal infections, such as pyelonephritis, endocarditis and meningitis, most often occur following hematogenous spread or in patients with anatomic abnormalities or implanted medical devices (e.g., prosthetic heart valves or central nervous system shunts). Widespread visceral dissemination occurs in patients with neutropenia when Candida species gain access to the bloodstream, and candidal infections can occur in various locations throughout the body; however, subcutaneous candidal abscess formation is very rare, even in immunocompromised patients. Some cases of subcutaneous candidal abscesses have been reported in patients with skin breakdown, such as that due to bacterial cellulitis or abscess formation, trauma, parenteral substance abuse, iatrogenic procedures or central venous catheter insertion (8).

This report describes the first reported instance of coinfection with $C$. albicans and $C r$. neoformans in a patient without human immunodeficiency virus (HIV)/AIDS infection and speculates an association with diabetes mellitus and steroid therapy.

\section{Case Report}

A 50-year-old man with type II diabetes (undergoing insulin treatment), hypertension and a herniated lumbar disc was diagnosed with Cushing's syndrome. Right adrenalectomy was performed two months before the described hospital admission, and daily corticosteroid replacement therapy (40 mg/day of hydrocortisone, $0.5 \mathrm{mg} /$ day of dexamethasone) was initiated. The patient had not received any antifungal or antimicrobial prophylaxis since the adrenalectomy. He was admitted to a local hospital due to lower leg palsy, suggesting exacerbation of the herniated lumbar disc. Chest radiography revealed a pulmonary nodule in the right upper field of the lung, and he was transferred to our hospital for a further examination.

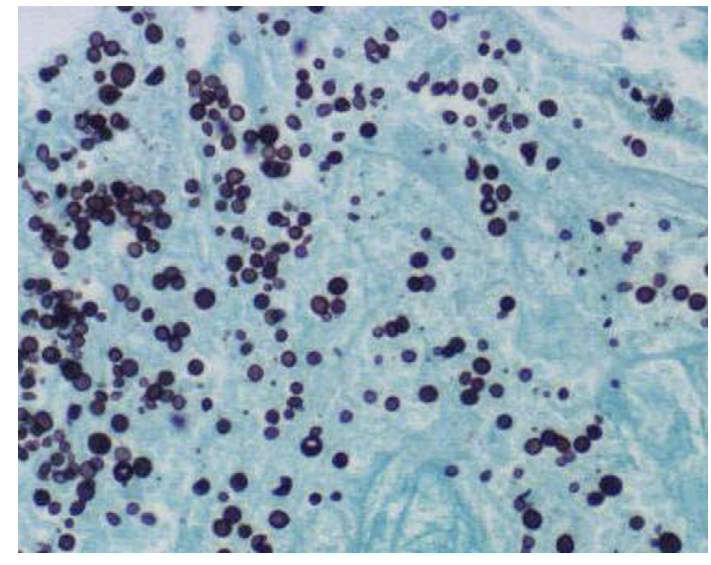

Figure 2. A histopathological examination of a lung biopsy specimen. Gomori's methenamine-silver stain showing encapsulated yeast forms that resemble $\mathrm{Cr}$. neoformans (Magnification, $\times 400$ ).

On admission, a physical examination revealed full moon face, centripetal obesity and mild pretibial pitting edema. No abnormal respiratory sounds were heard in either lung field, and no heart murmurs were audible. An abdominal examination showed abdominal striae, and a neurological examination revealed no nuchal rigidity, cranial nerve deficits or papilledema. The patient's tendon reflexes were normal without pathological reflexes; however, proximal muscle weakness was observed. His body temperature was $37.0^{\circ} \mathrm{C}$, his blood pressure was $140 / 91 \mathrm{mmHg}$ and his heart rate was 90 beats/min. The results of laboratory tests were as follows: leukocyte count, $13,200 / \mathrm{mm}^{3}$ (82\% polymorphonuclear leukocytes); hemoglobin level, $8.7 \mathrm{~g} / \mathrm{dL}$; hematocrit concentration, 30.8\%; serum Fe level, $42 \mu \mathrm{g} / \mathrm{dL}$ (suggesting iron deficiency anemia); platelet count, $645,000 / \mathrm{mm}^{3}$; urea level, 11 $\mathrm{mg} / \mathrm{dL}$; creatinine level, $0.63 \mathrm{mg} / \mathrm{dL}$; total protein level, 6.4 $\mathrm{g} / \mathrm{dL}$; albumin level, $3.8 \mathrm{~g} / \mathrm{dL}$; and C-reactive protein level, $0.10 \mathrm{mg} / \mathrm{dL}$. The CD4 count was $533 / \mu \mathrm{L}$. The fasting blood sugar level and HgbA1c concentration (Japan Diabetes Society) were $146 \mathrm{mg} / \mathrm{dL}$ and $7.7 \%$, respectively, suggesting poorly controlled diabetes mellitus. Regarding blood gases, the $\mathrm{PaO}_{2}$ and $\mathrm{PaCO}_{2}$ values were 59.2 and $50.7 \mathrm{mmHg}$ on room air, respectively. The cortisol level was $1.4 \mu \mathrm{g} / \mathrm{dL}$, and the adrenocorticotropic hormone level was $<5 \mathrm{pg} / \mathrm{mL}$. A serum cryptococcal antigen test was positive, with a titer of 1:8 (Serodirect $^{\circledR}$ "Eiken" Cryptococcus, Eiken Co., Tokyo, Japan), and the serum $\beta$-D-glucan level was $43.7 \mathrm{pg} / \mathrm{mL}$ (cutoff, $<20 \mathrm{pg} / \mathrm{mL}$; Fungitec G test, Seikagaku Kogyo, Tokyo, Japan). An HIV test was negative.

Thoracic computed tomography (CT) showed an 18-mm solitary, well-defined nodule in the right S2 region (Fig. 1). In order to examine the pulmonary nodule, a CT-guided biopsy was performed, followed by a histopathological examination of the lung biopsy specimen using Gomori's methenamine-silver stain, which showed encapsulated yeast forms that resembled $\mathrm{Cr}$. neoformans (Fig. 2). Although the patient had no headaches or nuchal rigidity, lumbar puncture 


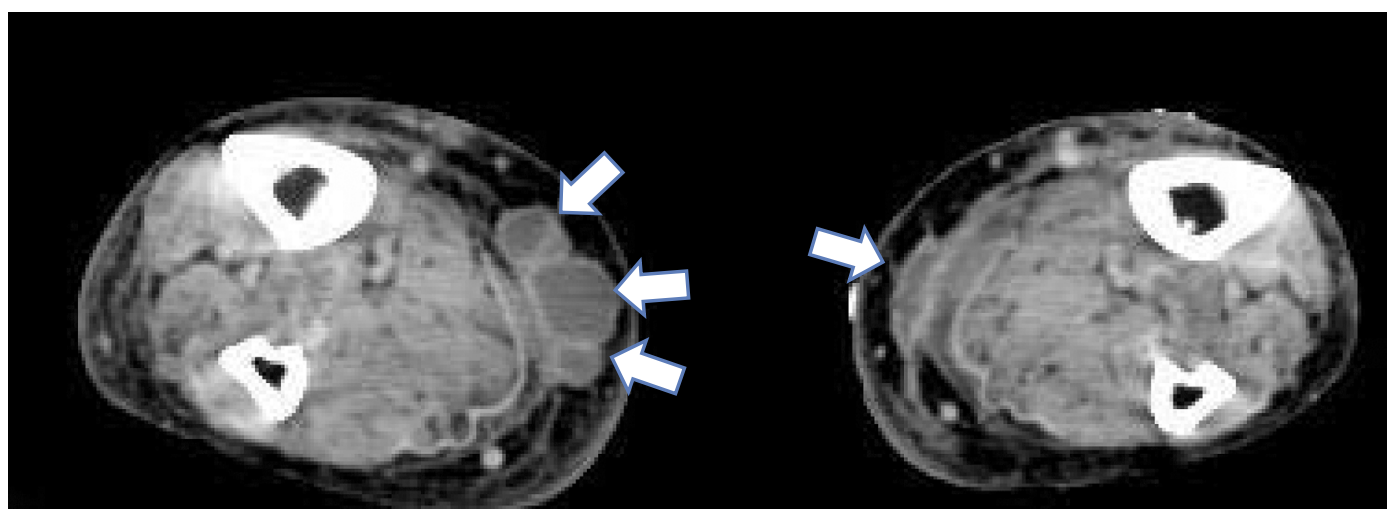

Figure 3. T1-weighted magnetic resonance image showing a well-demarcated collection of fluid in the soft tissue of both lower legs (arrows), suggesting a subcutaneous abscess.

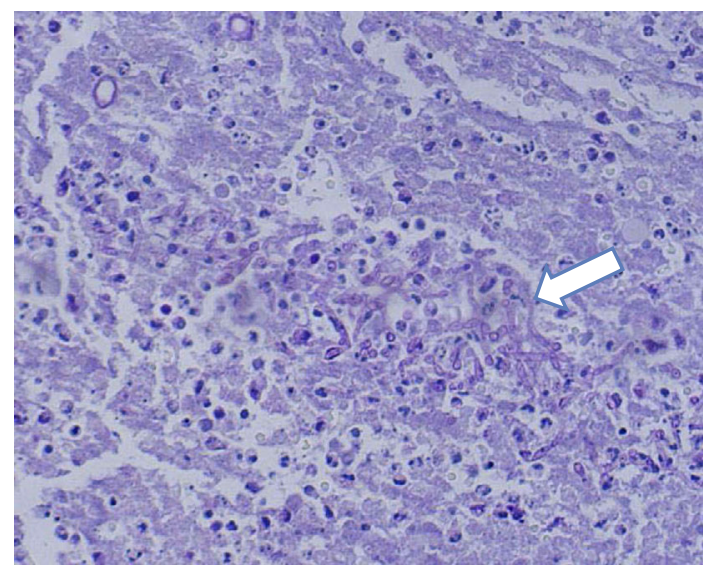

Figure 4. A histopathological examination of the needle aspirate of a lower extremity abscess. Periodic acid-Schiff stain showing yeast and hyphal forms (arrow) of the fungus (Magnification, $\times 400$ ).

was performed to confirm the diagnosis of cryptococcal meningoencephalitis. However, a cerebrospinal fluid cryptococcal antigen test was negative, the total nucleated cell count was not elevated and no Cr. neoformans was cultured. The patient was consequently diagnosed with pulmonary cryptococcosis based on the positive cryptococcal antigen test and pulmonary histopathological findings.

The patient subsequently developed several firm subcutaneous nodules on both lower legs. The nodules each measured approximately $1.0 \mathrm{~cm}$ in diameter and were reddish, movable and warm. A T1-weighted magnetic resonance image revealed a rounded fluid-collection signal in the soft tissue of the legs, suggesting a subcutaneous abscess formation (Fig. 3). The image showed no evidence of osteomyelitis. Needle aspiration was performed, and a histopathological examination using periodic acid-Schiff stain showed both the yeast and hyphal forms of the fungus (Fig. 4). C. albicans was successfully cultured from the aspirate. No bacteria were isolated from aerobic or anaerobic cultures, and a blood culture was negative. Diabetic retinopathy, without candidal endophthalmitis, was observed. No other signs of candidal infection (i.e., of the oral cavity, esophagus, nails or gastrointestinal tract) were observed.

The minimum inhibitory concentration (MIC) of the isolated $C$. albicans revealed that the pathogen was susceptible to all antifungals tested: voriconazole, 0.015; amphotericin B (AMPH-B), 0.125; flucytosine (5-FC), 0.25; fluconazole (FLCZ), 0.125; itraconazole, 0.03; and micafungin, $0.03 \mathrm{mg} /$ $\mathrm{mL}$. Therefore, the patient was treated with FLCZ (400 mg/ day) and 5-FC (8 g/day) for both fungal infections, and drainage of the subcutaneous abscesses was performed.

One month after the start of antifungal treatment, the size of the pulmonary cryptococcal nodule had generally decreased on thoracic CT, and the subcutaneous candidal abscesses had improved on lower extremity CT (Fig. 5A). In addition, the serum $\beta$-D-glucan level decreased to 19.6 and $27.1 \mathrm{ng} / \mathrm{mL}$ after one and three months, respectively.

Following a three-month course of FLCZ and 5-FC treatment, renal dysfunction was observed; therefore, both antifungals were stopped for a three-week period. Thereafter, only FLCZ was continued for a total of six months for treatment of pulmonary cryptococcosis according to the recommendations of the Japanese Mycology Study Group guidelines for patients with underlying disease (9). The titer of cryptococcal antigens gradually decreased over five months. After six months, the cryptococcal antigen test became negative and the pulmonary nodule reduced in size on thoracic CT (Fig. 5B).

The patient is currently receiving daily corticosteroid replacement therapy $(5 \mathrm{mg} /$ day of hydrocortisone and $0.5 \mathrm{mg}$ / day of dexamethasone) without antifungal treatment. Periodic follow-up observations have revealed no trends towards relapse.

\section{Discussion}

Subcutaneous candidal abscesses are very rare, even in immunocompromised patients (10). However, cases of such lesions have been reported in patients with skin breakdown, such as that due to bacterial cellulitis or abscess formation, iatrogenic procedures, trauma or parenteral substance 
(A)

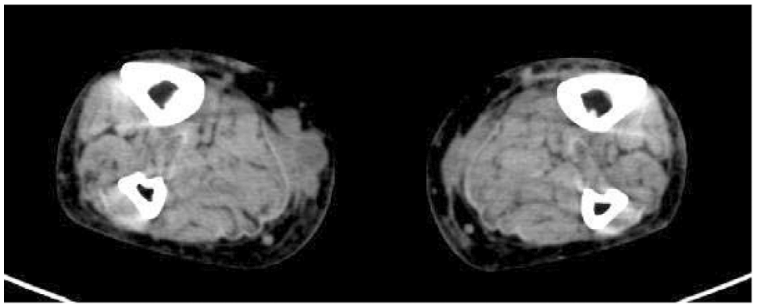

1 month later
(B)

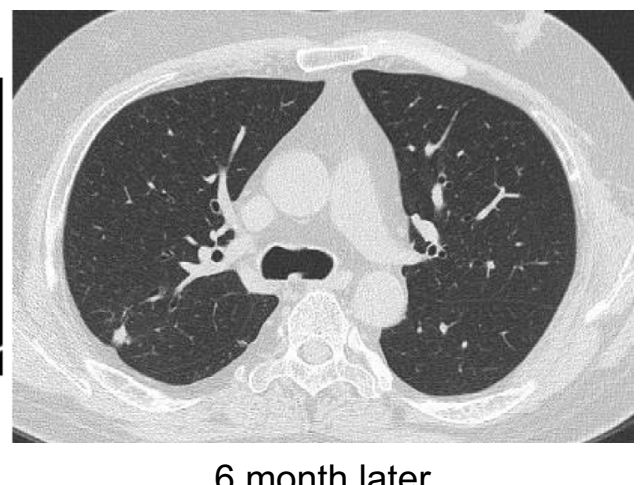

Figure 5. Antifungal therapy reduced the size of the patient's leg skin abscesses and lung nodule. (A) A leg CT image obtained one month after the initiation of antifungal therapy showing a reduction in the size of the skin abscesses. (B) A thoracic CT image obtained six months after the start of antifungal therapy showing a 5-mm (reduced from $18 \mathrm{~mm}$ ) nodule in the right $\mathrm{S} 2$ region.

abuse (11-16).

Many risk factors for the development of candidal infection have been recognized, including immunosuppression, corticosteroid use, chemotherapy, prolonged neutropenia, broad-spectrum antibiotic use, indwelling central catheter placement, hyperalimentation, dialysis, abdominal surgery disrupting the integrity of the bowel mucosa, intravenous drug use, prosthetic intravascular implantation, severe burns and Candida spp. colonization $(17,18)$. Florescu et al. reviewed the risk factors associated with the development of subcutaneous candidal abscesses and found that these factors included indwelling central venous (CV) and intravenous catheter placement, antibiotic use, diabetes mellitus, gastrointestinal surgery, hyperalimentation, immunosuppression due to corticosteroid therapy or AIDS and Candida spp. colonization (19). In the present case, the patient had several of the above mentioned factors, i.e., uncontrolled diabetes mellitus and corticosteroid use, due to which, he was at risk of candidal abscess infection. Several firm subcutaneous nodules were observed on both lower legs. However, no apparent injuries or scars were noted. Lower extremity CT showed subcutaneous abscesses on both legs, suggesting that Candida had disseminated via the bloodstream. Sites of intravenous catheter placement and the gastrointestinal tract are major portals of entry for systemic Candida infection. However, the patient did not have a CV catheter for a long period. Therefore, the gastrointestinal tract may have been the portal of entry in this case.

Although cryptococcal infection can develop in individuals with normal immunity, it most commonly occurs in immunocompromised hosts. Cell-mediated immunodeficiency is an important underlying condition of cryptococcal infection. Predisposing factors include AIDS and other causes of impaired T cell-mediated immunity, e.g., transplant-related immunosuppression, hematological malignancies, corticosteroid administration and diabetes mellitus (20-23). Prolonged, high-dose corticosteroid use (i.e., $20 \mathrm{mg} /$ day) is re- ported to be an independent factor for disseminated disease (24). In the present case, uncontrolled diabetes and corticosteroid use were co-factors of pulmonary cryptococcosis and candidal skin abscess formation.

Neutrophil chemotaxis and adherence to the vascular endothelium, phagocytosis, intracellular bactericidal activity, opsonization and cell-mediated immunity are all depressed in diabetes patients with hyperglycemia $(25,26)$. In addition, glucose-inducible proteins promote the adhesion of $C$. albicans to buccal or vaginal epithelium, which impairs phagocytosis, giving the organism an advantage over the host (27). Glucocorticoid administration also results in neutrophilic leukocytosis accompanied by dramatic reductions in circulating eosinophils, monocytes and lymphocytes (28). In the present case, CD4 lymphopenia was observed without HIV infection. CD4 lymphopenia is thought to be related to impaired T-cell-mediated immunity, resulting in disseminated candidiasis and cryptococcosis.

Little is known about other risk factors for the development of co-infection. TNF- $\alpha$ and interleukin (IL)-17 may be key factors for co-infection. TNF- $\alpha$ is a factor for the reduction of the pathogenic burden of $C$. albicans in animals $(29,30)$. In contrast, cryptococcal infection is reported to inhibit TNF- $\alpha$ production (31). Furthermore, cryptococcal infection may exacerbate candidiasis.

IL-17 is a proinflammatory cytokine produced by a subset of CD4 T-cells, termed Th17 cells. Increased IL-17 production is associated with a reduced cryptococcal burden, suggesting that IL-17 plays a significant role in the generation of a protective anti-cryptococcal immune response. In contrast, $C$. albicans is reported to dampen the host defense by downregulating IL-17 production (32). Candidal infection may alter cellular immunity and is recognized to be a predisposing factor for developing cryptococcosis. Altered host immunity may explain why cryptococcosis and candidiasis developed in this patient without HIV infection.

During a 35-year period at Nagasaki University hospital 
and its affiliates, the diagnosis of pulmonary cryptococcosis was confirmed in 151 patients. Of these patients, only the present subject exhibited pulmonary cryptococcosis and candidal skin abscess co-infection (0.66\%, unpublished data).

Approximately two decades have passed since the introduction of the serum $\beta$-D-glucan assay for the clinical diagnosis of deep-seated mycosis in Japan (33). The assay is now widely accepted in Japan and other countries as an indispensable tool for managing febrile episodes in immunocompromised hosts. In addition, its use is included in the guidelines for the diagnosis and treatment of deep-seated mycosis $(9,34,35)$. The presence of $\beta$-D-glucan in the serum signifies the presence of fungal invasion; however, the results are not specific for Candida species (36). Falsepositive findings can occur for a variety of reasons, including the use of glucan-contaminated blood collection tubes, gauze and depth-type membrane filters for blood processing, as well as in vitro tests using various antibiotics (e.g., some cephalosporins, carbapenems and ampicillinsulbactam) $(34,37)$. Therefore, a serum $\beta$-D-glucan level exceeding the cutoff value even slightly may indicate the absence of deep-seated mycoses. A high serum $\beta$-D-glucan level is associated with cryptococcal meningitis (38) and cryptococcemia (39). The present patient was diagnosed with pulmonary cryptococcosis in addition to a moderate immunosuppressive state. A lumbar puncture did not reveal cryptococcal meningoencephalitis, and no microorganisms were cultured from his blood. On admission, the serum $\beta$-Dglucan level was approximately twice the cutoff value. Therefore, we interpreted this to be a false-positive result. However, a cautious medical examination revealed that he had candidal skin abscess co-infection.

Cryptococcus was not cultured in this case; therefore, we were unable to perform antifungal susceptibility tests for Cryptococcus isolates. It is difficult to distinguish between $\mathrm{Cr}$. neoformans and $\mathrm{Cr}$. gattii infections based on the results of histopathological examinations. Nevertheless, the first case report of a patient in Japan infected with $\mathrm{Cr}$. gattii genotype VGIIa noted that the patient had no recent history of travel to any disease endemic areas (40), suggesting that the virulent strain may have spread to regions outside North America. Cr. gattii is generally geographically restricted. Furthermore, among cryptococcal infections in Japan, $C r$. neoformans (serotype A) is the most common, with a frequency exceeding 95\% (41). In addition, no azole-resistant Cr. neoformans isolates have been detected, even in the latest reports (42).

The $C$. albicans isolated from this patient was found to be susceptible to all antifungals tested. In the updated practical guidelines for the management of candidiasis issued by the Infection Disease Society of America, treatment with FLCZ or echinocandin is recommended for candidemia in patients with neutropenia as the initial therapy (43). The administration of a combination of the lipid formulation AMPH-B (LFAmB) or AMPH-B deoxycholate with 5-FC is recommended in cases of central nervous system (CNS) can- didiasis, candidal endophthalmitis, candidal infection of the cardiovascular system, endocarditis and others (43). We chose the combination of FLCZ and 5-FC as the initial antifungal therapy in the present case based on the antifungal activity against both $C r$. neoformans and $C$. albicans infection $(44,45)$. On the other hand, if a non-albicans Candida species e.g., C. glabrata or C. krusei, had been identified, these antifungal agents would have been changed to LFAmB. However, the patient's treatment was successful, as originally prescribed.

In conclusion, we herein reported, to the best of our knowledge, the first published case of pulmonary cryptococcosis and candidal skin abscess co-infection in an immunocompromised patient.

\section{Author's disclosure of potential Conflicts of Interest (COI).}

Shigeru Kohno: Honoraria, Consultation fee and Research funding, Pfizer Inc.

\section{Acknowledgement}

This work was partly supported by the Ministry of Health, Labour and Welfare Sciences Research Grants (H25-Shinko-ippan006) and a Grant-in-Aid for Scientific Research (C) from the Ministry of Education, Culture, Sports, Science and Technology of Japan (25461516).

\section{References}

1. Kovacs JA, Kovacs AA, Polis M, et al. Cryptococcosis in the acquired immunodeficiency syndrome. Ann Intern Med 103: 533538, 1985.

2. Powderly WG. Cryptococcal meningitis and AIDS. Clin Infect Dis 17: 837-842, 1993.

3. Ellis DH, Pfeiffer TJ. Natural habitat of Cryptococcus neoformans var. gattii. J Clin Microbiol 28: 1642-1644, 1990.

4. Pfeiffer TJ, Ellis DH. Environmental isolation of Cryptococcus neoformans var. gattii from Eucalyptus tereticornis. J Med Vet Mycol 30: 407-408, 1992.

5. Kidd SE, Hagen F, Tscharke RL, et al. A rare genotype of Cryptococcus gattii caused the cryptococcosis outbreak on Vancouver Island (British Columbia, Canada). Proc Natl Acad Sci USA 101: 17258-17263, 2004.

6. Abi-Said D, Anaissie E, Uzun O, Raad I, Pinzcowski H, Vartivarian S. The epidemiology of hematogenous candidiasis caused by different Candida species. Clin Infect Dis 24: 1122-1128, 1997.

7. Leroy O, Gangneux JP, Montravers P, et al. Epidemiology, management, and risk factors for death of invasive Candida infections in critical care: a multicenter, prospective, observational study in France (2005-2006). Crit Care Med 37: 1612-1618, 2009.

8. Mochizuki T, Urabe Y, Hirota Y, Watanabe S, Shiino A. A case of Candida albicans skin abscess associated with intravenous catheterization. Dermatologia 177: 115-119, 1988.

9. The Japanese Mycology Study Group. Guidelines for Deep-seated Mycoses in Japan 2007. Kyowa Kikaku Ltd., Tokyo, Japan, 2007: 8-12.

10. Champman SW, Daniel CR. Cutaneous manifestations of fungal infections. Infect Dis Clin N Am 8: 879-910, 1994.

11. Cuozzo DW, Aaronsin B, Benson PM, Sau P. Candida krusei abdominal wall abscess presenting as ecchymosis. Diagnosis with ultrasound. Arch Derm 131: 275-277, 1995.

12. Ginter G, Rieger E, Soyer HP, Hoedl S. Granulomatous panniculitis caused by Candida albicans: a case presenting with multiple 
leg ulcers. J Am Acad Dermatol 28: 315-317, 1993.

13. Manfredi R, Mazzoni A, Nanetti A, Mastroianni A, Coronado OV, Chiodo F. Isolated subcutaneous candidal abscess and HIV disease. Br J Dermatol 136: 647-649, 1997.

14. Mazumdar PK, Marks MI. Candida albicans infections in hospitalized children: a survey of predisposing factors. Clin Pediatr (Phila) 14: 123-129, 1975.

15. Shiota T, Ikeda S, Konishi T, et al. Mediastinitis and left pyopneumothorax complicating a laryngeal phlegmon. Nippon Kyobu Shikkan Gakkai Zasshi 27: 1367-1370, 1989.

16. Szilagyi G, Reiss F. Fungus infections at Montefiore Hospital and Medical Center. NY St J Med 66: 3036-3039, 1966.

17. Bennett JE. Introduction to mycoses. In: Principles and Practices of Infectious Diseases. 6th ed. Mandel GL, Bennett JE, Dolin R, Eds. Elsevier Churchill Livingstone, Philadelphia, 2005: 29352951.

18. Laupland KB, Gregson DB, Church DL, Ross T, Elsayed S. Invasive Candida species infections: a 5-year population-based assessment. J Antimicrob Chemother 56: 532-537, 2005.

19. Florescu DF, Brostrom SE, Dumitru I, Kalil AC. Candida albicans skin abscess in a heart transplant recipient. Infect Dis Clin Pract 18: 243-246, 2010.

20. Woodring JH, Ciporkin G, Lee C, Worm B, Woolley S. Pulmonary cryptococcosis. Semin Roentgenol 31: 67-75, 1996.

21. Kerkering TM, Duma RJ, Shadomy S. The evolution of pulmonary cryptococcosis: clinical implications from a study of $41 \mathrm{pa}-$ tients with and without compromising host factors. Ann Intern Med 94: 611-616, 1981.

22. Rozenbaum R, Goncalves AJ. Clinical epidemiological study of 171 cases of cryptococcosis. Clin Infect Dis 18: 369-380, 1994.

23. Perfect JR. Cryptococcosis. Infect Dis Clin N Am 3: 77-102, 1989.

24. Baddley JW, Perfect JR, Oster RA, et al. Pulmonary cryptococcosis in patients without HIV infection: factors associated with disseminated disease. Eur J Clin Microbiol Infect Dis 27: 937-943, 2008.

25. Delamaire M, Maugendre D, Moreno M, et al. Impaired leucocyte functions in diabetic patients. Diabet Med 14: 29-34, 1997.

26. Llorente L, De La Fuente H, Richaud-Patin Y, et al. Innate immune response mechanisms in non-insulin dependent diabetes mellitus patients assessed by flow cytoenzymology. Immunol Lett 74: 239-244, 2000.

27. Hostetter MK. Handicaps to host defense. Effects of hyperglycemia on C3 and Candida albicans. Diabetes 39: 271-275, 1990.

28. Fauci AS, Dale DC, Balow JE. Glucocorticosteroid therapy: mechanisms of action and clinical considerations. Ann Intern Med 84: 304-315, 1976.

29. Djeu J, Blanchard D, Halkis D, Friedman H. Growth inhibition of Candida albicans by human polymorphonuclear neutrophils: activation by interferon-gamma and tumor necrosis factor. J Immunol 137: 2980-2984, 1986.

30. Ferrante A. Tumor necrosis factor alpha potentiates neutrophil antimicrobial activity: increased fungicidal activity against Torulopsis glabrata and Candida albicans and associated increases in oxygenradical production and lysosomal enzyme release. Infect Immun 57: 2115-2122, 1989.

31. Huffnagle GB, Chen GH, Curtis JL, McDonald RA, Strieter RM,
Toews GB. Down-regulation of the afferent phase of $\mathrm{T}$ cellmediated pulmonary inflammation and immunity by a high melanin-producing strain of Cryptococcus neoformans. J Immunol 155: 3507-3516, 1995.

32. Cheng SC, Veerdonk F, Smmekens S, et al. C. albicans dampens host defense by downlegulating IL-17 production. J Immunol 185: 2450-2457, 2010.

33. Obayashi $\mathrm{T}$, Yoshida $\mathrm{M}$, Mori $\mathrm{T}$, et al. Plasma (1,3)- $\beta$-D-glucan measurement in diagnosis of invasive deep mycosis and fungal febrile episodes. Lancet 345: 17-20, 1995.

34. Walsh TJ, Anaissie EJ, Denning DW, et al. Treatment of aspergillosis: clinical practice guidelines of the Infectious Diseases Society of America. Clin Infect Dis 46: 327-360, 2008.

35. Freifeld AG, Bow EJ, Sepkowitz KA, et al. Clinical practice guideline for the use of antimicrobial agents in neutropenic patients with cancer: 2010 update by the Infectious Diseases Society of America. Clin Infect Dis 52: 56-93, 2011.

36. Pickering JW, Sant HW, Bowles CA, Roberts WL, Woods GL. Evaluation of a $(1 \rightarrow 3)$ - $\beta$-D-glucan assay for diagnosis of invasive fungal infections. J Clin Microbiol 43: 5957-5962, 2005.

37. Marty FM, Lowry CM, Lempitski SJ, Kubiak DW, Finkelman MA, Baden LR. Reactivity of ( $1 \rightarrow 3)-\beta$-D-glucan assay with commonly used intravenous antimicrobials. Antimicrob Agents Chemother 50: 3450-3453, 2006.

38. Obayashi $T$, Yoshida $M$, Tamura $H$, Aketagawa J, Tanaka $S$, Kawai T. Determination of plasma $(1 \rightarrow 3)-\beta-D-$ glucan: a new diagnostic aid to deep mycosis. J Med Vet Mycol 30: 275-280, 1992.

39. Obayashi T, Negishi K, Suzuki T, Funata N. Reappraisal of the serum $(1 \rightarrow 3)-\beta-D-$ glucan assay for the diagnosis of invasive fungal infections: a study based on autopsy cases from 6 years. Clin Infect Dis 46: 1864-1870, 2008

40. Okamoto K, Hatakeyama S, Itoyama S, et al. Cryptococcus gattii genotype VGIIa infection in man, Japan, 2007. Emerg Infect Dis 16: 1155-1157, 2010.

41. Kohno S, Varma A, Kwon-Chung KJ, Hara K. Epidemiology studies of clinical isolates of Cryptococcus neoformans of Japan by restriction fragment length polymorphism. Kansenshogaku Zasshi 68: 1512-1517, 1994.

42. Pfaller MA, Messer SA, Woosley LN, Jones RN, Castanheira M. Echinocandin and triazole antifungal susceptibility profiles for clinical opportunistic yeast and mold isolates collected from 2010 to 2011: application of new CLSI clinical breakpoints and epidemiological cutoff values for characterization of geographic and temporal trends of antifungal resistance. J Clin Microbiol 51: 2571-2581, 2013.

43. Pappas PG, Kauffman CA, Andes D, et al. Clinical practice guidelines for the management of candidiasis: 2009 update by the Infectious Diseases Society of America. Clin Infect Dis 48: 503-535, 2009.

44. Nussbaum JC, Jackson A, Namarika D, et al. Combination flucytosine and high-dose fluconazole compared with fluconazole monotherapy for the treatment of cryptococcal meningitis: a randomized trial in Malawi. Clin Infect Dis 50: 338-344, 2010.

45. Scheven M, Jünemann K, Schramm H, Hühn W. Successful treatment of a Candida albicans sepsis with a combination of flucytosine and fluconazole. Mycoses 35: 315-316, 1992.

(C) 2014 The Japanese Society of Internal Medicine

http://www.naika.or.jp/imonline/index.html 